\title{
Single-Session treatment of Upper Extremity Deep Venous Thrombosis and Central Venous Catheter Malfunction using INARI ClotTriever
}

Siddharth Agarwal MBBS', Christopher Sosnofsky DO², Jamie Saum RN³, Manu B Aggarwal $\mathrm{MD}^{4}$, Sandeep M. Patel MD $\mathrm{M}^{3}$

Affiliations:

${ }^{1}$ Vardhman Mahavir Medical College and Safdarjung Hospital. New Delhi, India

${ }^{2}$ Heritage College of Osteopathic Medicine, Athens, Ohio

${ }^{3}$ Structural Heart and Intervention Center, St. Rita's Medical Center, Lima, OH

${ }^{4}$ Vein Care Center, Lima, OH

Affiliations: None

Address for Correspondence:

Sandeep M Patel, MD, FACC, FSCAI, RPVI 
Director, Structural Heart and Intervention Center

St. Rita's Medical Center (Mercy)

730 West Market Street, 2K Tower

Lima, Ohio 45801

Phone: (419) 996-5756

Fax: (419) 996-5854

Email: SMPatel@mercy.com

\title{
Acknowledgement: None
}

\begin{abstract}
UEDVT still remains an underdiagnosed entity due to a significant percentage of patients who remain asymptomatic. The etiology of UEDVT is overwhelmingly attributed to secondary causes including but not limited to central venous access devices and malignancy. Treatment modalities include anticoagulation, catheter directed thrombolysis, percutaneous mechanical thrombectomy or a multimodality approach along with the management of underlying etiology. We describe a case of a female with breast cancer, who came with left UEDVT and CVC malfunction and was successfully treated using the ClotTriever system to maintain continuous intravenous access.
\end{abstract}


Keywords:

Percutaneous mechanical thrombectomy

ClotTriever

Upper extremity deep venous thrombosis

Central venous catheter

Conflicts of Interests: None

Disclosure: None

Consent: written consent has been taken from the patient regarding the publication of case details as well as the images.

Funding: None 


\section{Introduction}

Intravenous catheters account for majority of the cases of upper extremity deep vein thrombosis (UEDVT), with even higher incidence in patients suffering from malignancy. Sequelae of UEDVT are similar to that of lower extremity DVT comprising of post thrombotic syndrome and pulmonary embolism (PE). Although the treatment modalities range from systemic anti-coagulation to catheter directed thrombolysis (CDT), percutaneous mechanical thrombectomy (PMT) and pharmacomechanical catheter directed thrombolysis (PMDT), due to the absence of consistent guidelines regarding the management of UEDVT, treatment is mostly individualized based on patient characteristics, clinical factors as well as technical considerations. We present a case of 49-year-old female suffering from breast cancer with a central venous catheter (CVC) who came to the clinic with UEDVT and CVC malfunction and was successfully treated with mechanical thrombectomy using ClotTriever system (Inari Medical, Irvine, CA).

\section{Case Description}

A 49-year-old female suffering from right sided triple negative breast cancer, on active chemotherapy, presented to the clinic with left arm swelling for the past 10 days. She states that her left arm started to swell which progressed from the elbow up to the shoulder. She also complained of heaviness and cramping pain in the left arm, $5 \backslash 10$ in severity which progressed to the extent of her inability to lift the 
left arm. Physical examination was remarkable for 4+ edema and tenderness along with plethora at the level of the shoulder and upper left chest. She was not able to lift her hand above the shoulder and also had restricted range of motion of the left elbow, due to swelling. She also had a left sided CVC which on further assessment was unable to be flushed or aspirated.

The patient was admitted and preliminary investigations performed were remarkable for anemia (7.8 gmldl) and leukopenia $\left(3,200 \backslash \mathrm{mm}^{3}\right)$. In view of catheter associated UEDVT, venous duplex ultrasonography was performed which showed acute occlusive DVT of the left subclavian and axillary vein along with superficial venous thrombosis of the proximal left basilic and antecubital veins (Fig 1).

As the diagnosis of catheter associated UEDVT was confirmed, the patient was started on subcutaneous enoxaparin injection $(1 \mathrm{mg} / \mathrm{kg})$ every 12 hours and the interventional cardiology service was consulted to discuss the need for possible intervention. Considering the patient's clinical as well as imaging presentation including severely progressive symptoms, high thrombotic burden extending from subclavian to axillary vein, along with the need to treat CVC malfunction and presence of severe anemia, decision was made to use ClotTriever system.

Left brachial 5-French venous access was obtained and diagnostic venography was performed and thrombotic occlusion of the brachial, axillary and subclavian vein was seen along with collateralization towards the SVC and presence of a large thrombus at the location of the CVC extending into the subclavian vein (Fig 2).

Given the abovementioned findings, the occlusion was crossed placing the wire in the SVC and further navigated into the IVC through the right atrium. This was then exchanged to an Amplatz Super Stiff wire that was placed in the right femoral vein and the Inari ClotTriever 13Fr sheath was then inserted into the left brachial vein after pre-insertion venoplasty with a $6.0 \mathrm{~mm}$ balloon. The Inari ClotTriever 
device was then inserted beyond the SVC and the catheter was deployed and after two passes using the ClotTriever device, repeat venography was performed which demonstrated $>90 \%$ patency of the brachial, axillary and subclavian veins along with minimal clot burden and no significant collaterals and straight-line flow to the SVC (Fig 4).

Overnight the patient was monitored in the cardiac telemetry unit and was discharged the next day on full dose subcutaneous enoxaparin with scheduled follow up after 1 week. On the follow up visit, the patient had significant improvement in the symptoms along with resolution of venous engorgement without any sign of bleeding.

\section{Discussion}

According to Otten et al., the incidence of UEDVT due to central catheters was found to be 6 per 10,000 hospital admissions; in fact, patients who have underlying malignancy have even higher incidence of approximately $3.8 \%$ in 12 months (1).

Although the incidence of PE is considered to be lower with UEDVT than with LEDVT, a recent study found the incidence of PE to be as high as $15 \%$ in ICU patients with UEDVT compared to $8 \%$ associated with LEDVT (2).

The goals of management of catheter associated UEDVT include alleviation of symptoms, prevention of embolization, along with maintenance of continuous intravenous access. In the absence of contraindications, systemic anticoagulation underpins all the treatment modalities, with low-molecular weight heparin being the preferred choice for patients with malignancy. Although the American College of Chest Physicians guidelines recommend anticoagulation over invasive management, interventional 
therapies should be considered in patients with severe progressive symptoms, thrombus extending from subclavian to axillary vein, symptoms $<14$ days, life expectancy $>1$ year, of the need for continued intravenous access and when appropriate technological expertise is available (3). Thrombus removal can be achieved through a catheter directed thrombolysis (CDT), percutaneous mechanical thrombectomy (PMT) or pharmaco-mechanical catheter directed thrombolysis. However, although using PCDT led to > $50 \%$ reduction in clot burden in all the patients (4), a major drawback of thrombolysis is that it is associated with clinically significant bleeding. Additionally, the use of CDT requires extended hospital stay in the ICU as well as serial venography which confers extra hospital costs as well as increased contrast burden on the kidneys respectively, which again should be an important consideration in patients already on chemotherapy. As in our patient who had severe progressive symptoms, long life expectancy, acute presentation, requirement of long-term CVC along with severe anemia, PMT using ClotTriever was a novel as well as the most appropriate treatment option. Although other PMT are available, ClotTriever was chosen due to the large burden of thrombus without needing thrombolysis.

Also imperative to this discussion is the need to maintain continuous intravenous access especially in patients with malignancy who require long-term chemotherapy as they have a higher incidence of catheter occlusion and resulting malfunction due to formation of "fibrin sheath" which might require removal and reinsertion of catheter which has its own technical difficulties along with higher chances of re-thrombosis. Although use of fibrinolytic agents has been used to dissolve the fibrin sheath, it does not clear the vein of thrombus thus it represents a temporary solution to allow for the use of the CVC but does not improve venous patency and associated patient symptoms., The Inari ClotTriever provides a safe, effective, nonthrombolytic option that can also be used for salvaging CVC thereby preventing serious bleeding complications and simultaneously preventing extraction and reimplantation of the CVC. 


\section{Conclusion}

Even after increasing awareness, UEDVT still remains an underdiagnosed clinical entity with no proper multicenter randomized clinical trials or guidelines addressing treatment of this condition. Although anticoagulation remains the cornerstone of therapy, interventional therapies without use of thrombolytic agent appear to be safe and effective as a first line treatment especially in patients who require rapid treatment along with long term intravenous access and simultaneously have contraindications to thrombolysis. Our successful outcome using the ClotTriever device in our patient who presented with UEDVT and CVC malfunction further advocates the use of this treatment modality in these settings as a single session interventional procedure. 


\section{References}

1. Hervé Decousus, Aurélie Bourmaud, Pierre Fournel, Laurent Bertoletti, Carine Labruyère, Emilie Presles et al. Cancer-associated thrombosis in patients with implanted ports: a prospective multicenter French cohort study (ONCOCIP). Blood 2018; 132: 707-716.

2. Francois Lamontagne, Lauralyn McIntyre, Peter Dodek, Diane Heels-Ansdell, Maureen Meade, Julia Pemberton et al. Nonleg venous thrombosis in critically ill adults: a nested prospective cohort study. JAMA Intern Med. 2014;174(5):689-696.

3. Clive Kearon, Elie A Akl, Joseph Ornelas, Allen Blaivas, David Jimenez, Henri Bounameaux et al. Antithrombotic Therapy for VTE Disease: CHEST Guideline and Expert Panel Report. Chest 2016; 149: 315-352.

4. Chaudhry MA, Pappy R, Hennebry TA. Use of the trellis device in the management of deep vein thrombosis: a retrospective single-center experience. J Invasive Cardiol 2013; 25: 296-299. 


\section{Figures}

Figure 1: Pre-procedure venous doppler ultrasonography showing absence of flow in the subclavian vein

Figure 2: Pre-procedure venography showing thrombosis of subclavian, axillary and brachial vein and presence of collaterals.

Figure 3: Post-procedure venography showing patent subclavian, axillary and brachial vein along with resolution of collaterals. 
Figure 1:

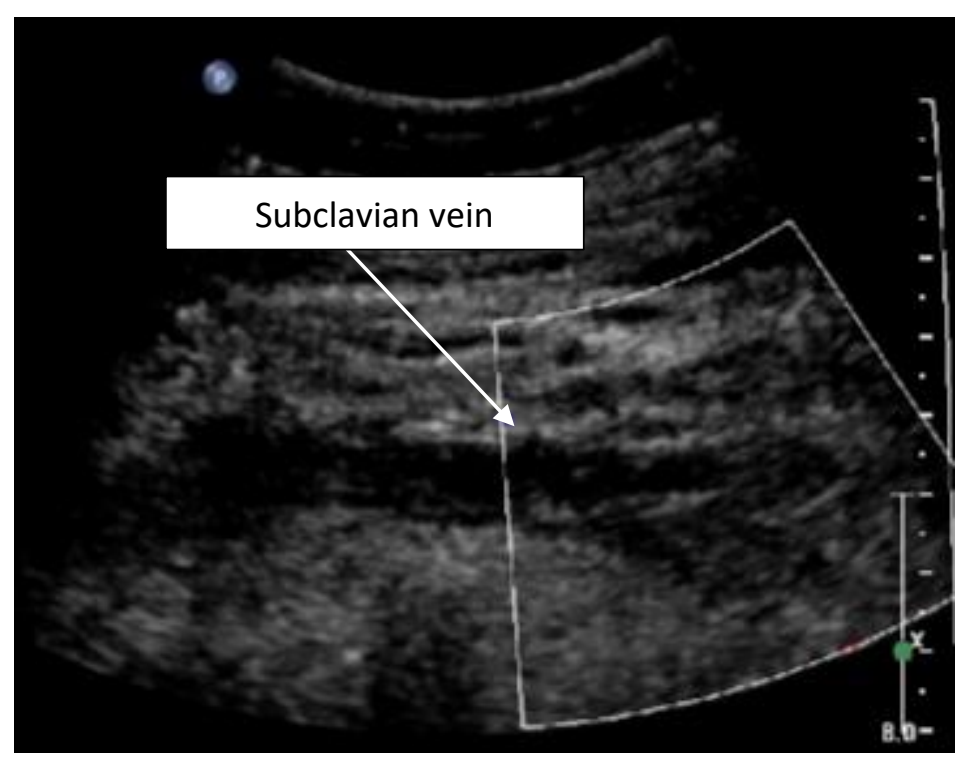




\section{Figure 2:}

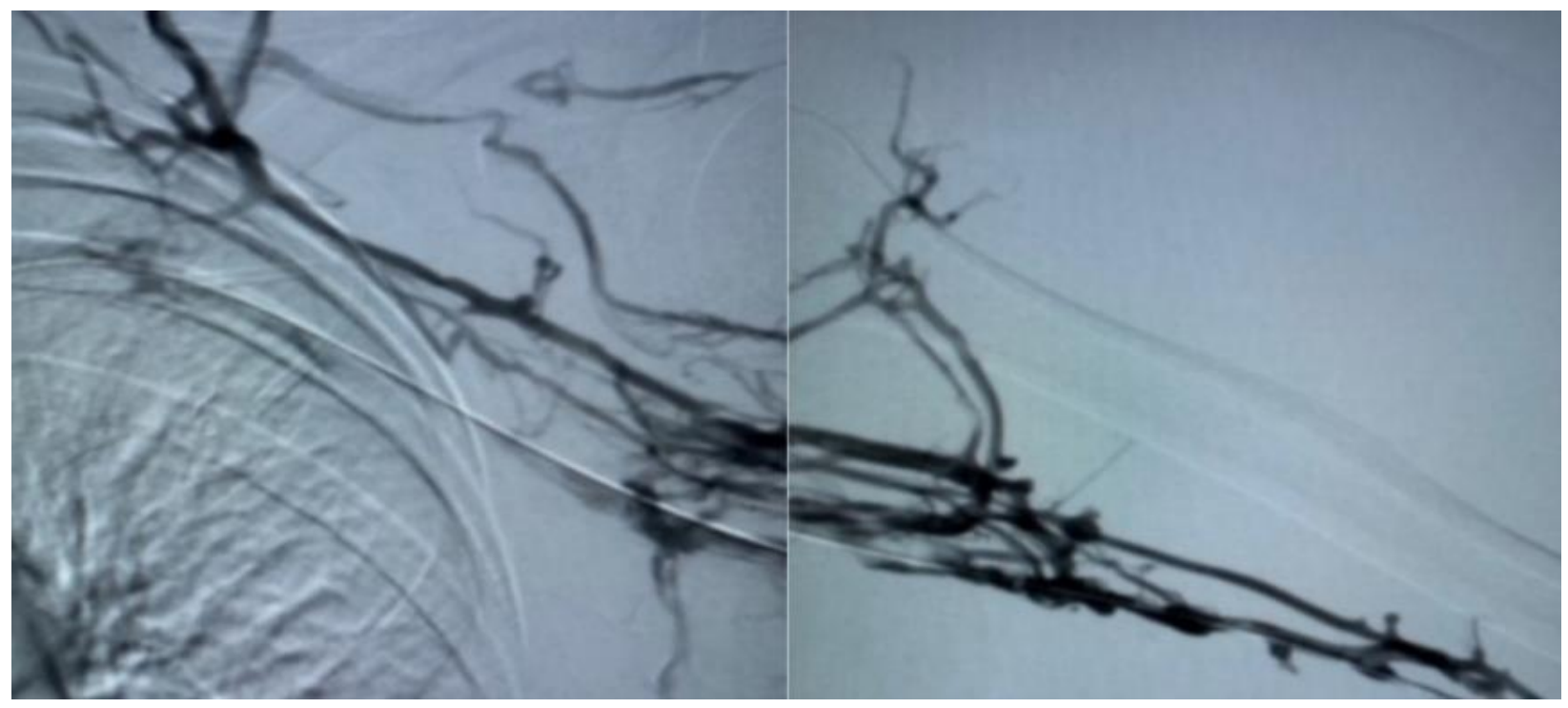




\section{Figure 3:}

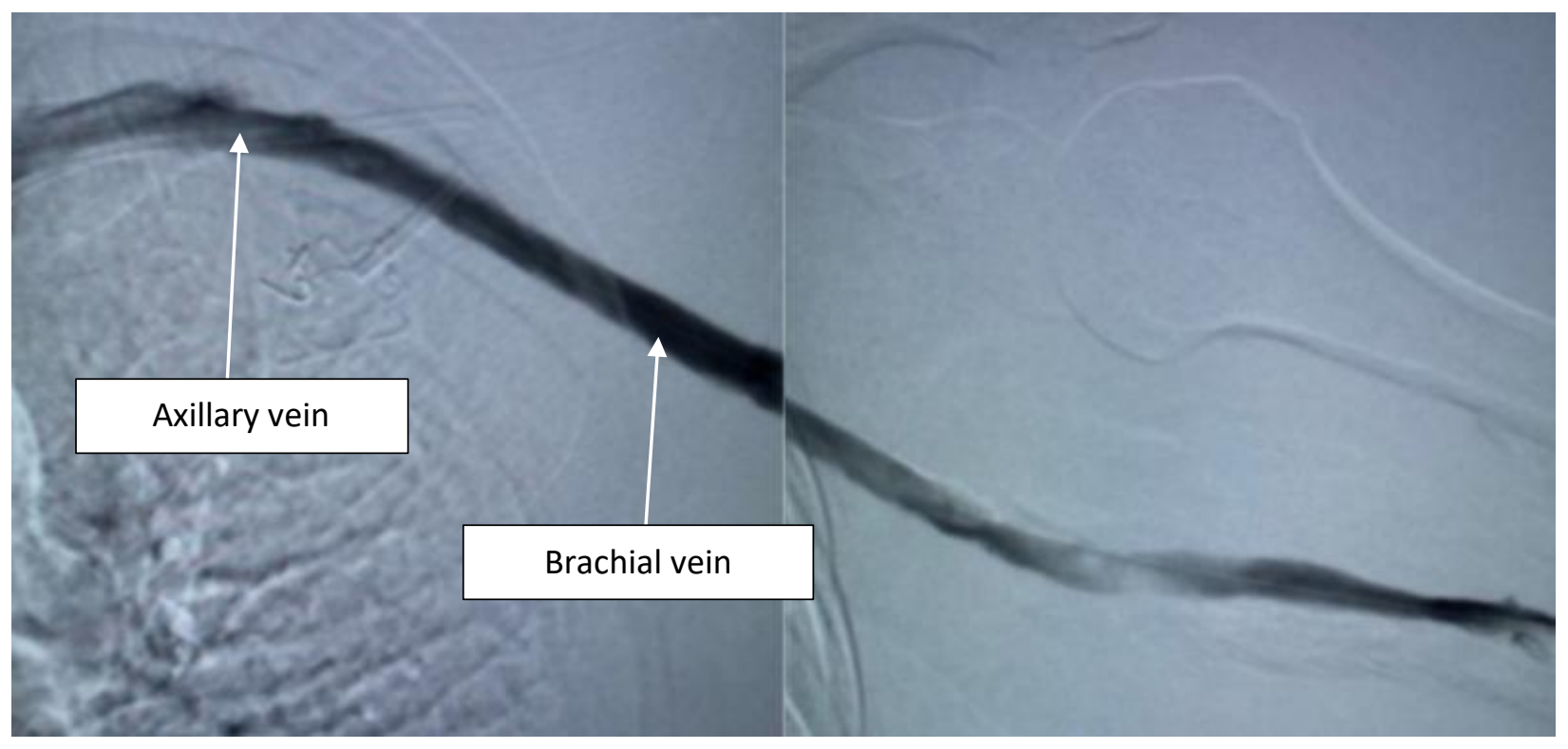


